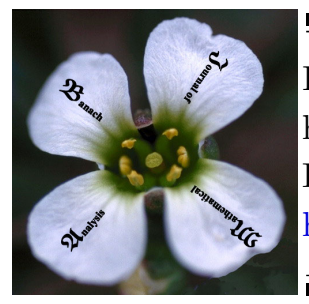

Banach J. Math. Anal. 9 (2015), no. 2, 127-133

http://doi.org/10.15352/bjma/09-2-10

ISSN: $1735-8787$ (electronic)

http://projecteuclid.org/bjma

\title{
ON THE SPECTRAL RADIUS OF HADAMARD PRODUCTS OF NONNEGATIVE MATRICES
}

\author{
DONGJUN CHEN AND YUN ZHANG*
}

Communicated by F. Zhang

\begin{abstract}
We present some spectral radius inequalities for nonnegative matrices. Using the ideas of Audenaert, we then prove the inequality which may be regarded as a Cauchy-Schwarz inequality for spectral radius of nonnegative matrices

$$
\rho(A \circ B) \leq[\rho(A \circ A)]^{\frac{1}{2}}[\rho(B \circ B)]^{\frac{1}{2}} .
$$

In addition, new proofs of some related results due to Horn and Zhang, Huang are also given. Finally, we interpolate Huang's inequality by proving

$$
\begin{aligned}
\rho\left(A_{1} \circ A_{2} \circ \cdots \circ A_{k}\right) & \leq\left[\rho\left(A_{1} A_{2} \cdots A_{k}\right)\right]^{1-\frac{2}{k}}\left[\rho\left(\left(A_{1} \circ A_{1}\right) \cdots\left(A_{k} \circ A_{k}\right)\right]^{\frac{1}{k}}\right. \\
& \leq \rho\left(A_{1} A_{2} \cdots A_{k}\right) .
\end{aligned}
$$
\end{abstract}

\section{INTRODUCTION AND PRELIMINARIES}

Let $M_{n}$ denote the set of complex matrices of order $n$. For matrices $A=$ $\left(a_{i j}\right), B=\left(b_{i j}\right) \in M_{n}$, we denote by $\rho(A)$ the spectral radius of $A$, by $A \circ B=$ $\left(a_{i j} b_{i j}\right)$ the Hadamard product of $A$ and $B$. The notation $A \leq B$ means that $B-A$ is entrywise nonnegative.

Zhan [10] conjectured that $\rho(A \circ B) \leq \rho(A B)$ for nonnegative matrices $A, B \in$ $M_{n}$. This conjecture was confirmed by Audenaert in [1] by proving

$$
\rho(A \circ B) \leq \rho^{\frac{1}{2}}((A \circ A)(B \circ B)) \leq \rho(A B) .
$$

Date: Received: Mar. 25, 2014; Revised: Jun. 19, 2014; Accepted: Jun. 29, 2014.

* Corresponding author.

2010 Mathematics Subject Classification. Primary 15A18, 15B48; Secondary 47A10, 47B37.

Key words and phrases. Hadamard product, nonnegative matrices, spectral radius. 
These inequalities were established via a trace description of the spectral radius. Using the fact that the Hadamard product of two matrices is a principal submatrix of the Kronecker product, Horn and Zhang proved [5] the inequalities

$$
\rho(A \circ B) \leq \rho^{\frac{1}{2}}(A B \circ B A) \leq \rho(A B) .
$$

Huang [6] generalized the inequality $\rho(A \circ B) \leq \rho(A B)$ to an arbitrary number of nonnegative matrices:

$$
\rho\left(A_{1} \circ A_{2} \circ \cdots \circ A_{k}\right) \leq \rho\left(A_{1} A_{2} \cdots A_{k}\right) .
$$

Recently, Peperko [8] proved the inequalities for nonnegative matrices $A, B$,

$$
\rho(A \circ B) \leq \rho^{\frac{1}{2}}(A B \circ B A) \leq \rho^{\frac{1}{4}}(A B \circ A B) \rho^{\frac{1}{4}}(B A \circ B A) \leq \rho(A B) .
$$

The paper is organized as follows. Using the similar idea due to Peperko, we first give a new proof for (1.3). We then prove the inequality which may be regarded as a Cauchy-Schwarz inequality for spectral radius of nonnegative matrices

$$
\rho(A \circ B) \leq[\rho(A \circ A)]^{\frac{1}{2}}[\rho(B \circ B)]^{\frac{1}{2}} .
$$

In addition, new proofs of some related results due to Horn and Zhang, Huang are also given. Finally, we interpolate Huang's inequality by proving

$$
\begin{aligned}
\rho\left(A_{1} \circ A_{2} \circ \cdots \circ A_{k}\right) & \leq\left[\rho\left(A_{1} A_{2} \cdots A_{k}\right)\right]^{1-\frac{2}{k}}\left[\rho\left(\left(A_{1} \circ A_{1}\right)\left(A_{2} \circ A_{2}\right) \cdots\left(A_{k} \circ A_{k}\right)\right]^{\frac{1}{k}}\right. \\
& \leq \rho\left(A_{1} A_{2} \cdots A_{k}\right) .
\end{aligned}
$$

Note that the inequality (1.1) corresponds to the case $k=2$ of the above inequalities.

\section{Main Results}

The two inequalities in the following lemma are classical and can be found in $[4,11]$.

Lemma 2.1. Let $A, B \in M_{n}$ be nonnegative matrices.

(1) If $A \leq B$, then $\rho(A) \leq \rho(B)$.

(2) $\rho(A \circ B) \leq \rho(A) \rho(B)$.

The following result appeared in $[2,7,8]$. A new and straightforward proof is presented. The similar manner can be found in [9].

Lemma 2.2. Let $\left\{A_{1 m}\right\}_{m=1}^{t},\left\{A_{2 m}\right\}_{m=1}^{t}, \ldots,\left\{A_{s m}\right\}_{m=1}^{t}$ be nonnegative matrices of order $n$. Then

$$
\begin{gathered}
\left(A_{11} \circ A_{21} \circ \cdots \circ A_{s 1}\right)\left(A_{12} \circ A_{22} \circ \cdots \circ A_{s 2}\right) \cdots\left(A_{1 t} \circ A_{2 t} \circ \cdots \circ A_{s t}\right) \\
\leq\left(A_{11} A_{12} \cdots A_{1 t}\right) \circ\left(A_{21} A_{22} \cdots A_{2 t}\right) \circ \cdots \circ\left(A_{s 1} A_{s 2} \cdots A_{s t}\right) .
\end{gathered}
$$

Proof. For $p=1, \ldots, s, q=1, \ldots, t$, we denote by $a_{i j}^{(p q)}$ the entry of the matrix $A_{p q}$ in the position $(i, j)$. The $(i, j)$-th entry of

$$
\left(A_{11} \circ A_{21} \circ \cdots \circ A_{s 1}\right)\left(A_{12} \circ A_{22} \circ \cdots \circ A_{s 2}\right) \cdots\left(A_{1 t} \circ A_{2 t} \circ \cdots \circ A_{s t}\right)
$$


equals

$$
\sum_{j_{1}, j_{2}, \ldots, j_{t-1}}\left(a_{i j_{1}}^{(11)} a_{i j_{1}}^{(21)} \cdots a_{i j_{1}}^{(s 1)}\right)\left(a_{j_{1} j_{2}}^{(12)} a_{j_{1} j_{2}}^{(22)} \cdots a_{j_{1} j_{2}}^{(s 2)}\right) \cdots\left(a_{j_{t-1} j}^{(1 t)} a_{j_{t-1} j}^{(2 t)} \cdots a_{j_{1} j}^{(s t)}\right) .
$$

Denote

$$
L=\sum_{j_{1}, j_{2}, \ldots, j_{t-1}}\left(a_{i j_{1}}^{(11)} a_{i j_{1}}^{(21)} \cdots a_{i j_{1}}^{(s 1)}\right)\left(a_{j_{1} j_{2}}^{(12)} a_{j_{1} j_{2}}^{(22)} \cdots a_{j_{1} j_{2}}^{(s 2)} \cdots\left(a_{j_{t-1} j}^{(1 t)} a_{j_{t-1} j}^{(2 t)} \cdots a_{j_{1} j}^{(s t)}\right) .\right.
$$

Then

$$
\begin{aligned}
L & =\sum_{j_{1}, j_{2}, \ldots, j_{t-1}}\left(a_{i j_{1}}^{(11)} a_{j_{1} j_{2}}^{(12)} \cdots a_{j_{t-1} j}^{(1 t)}\right)\left(a_{i j_{1}}^{(21)} a_{j_{1} j_{2}}^{(22)} \cdots a_{j_{t-1} j}^{(2 t)} \cdots\left(a_{i j_{1}}^{(s 1)} a_{j_{1} j_{2}}^{(s 2)} \cdots a_{j_{t-1} j}^{(s t)}\right)\right. \\
& \leq \sum_{j_{1}, j_{2}, \ldots, j_{t-1}}\left(a_{i j_{1}}^{(11)} a_{j_{1} j_{2}}^{(12)} \cdots a_{j_{t-1} j}^{(1 t)}\right) \cdots \sum_{j_{1}, j_{2}, \ldots, j_{t-1}}\left(a_{i j_{1}}^{(s 1)} a_{j_{1} j_{2}}^{(s 2)} \cdots a_{j_{t-1} j}^{(s t)}\right) .
\end{aligned}
$$

Note that for $p=1, \ldots, s$, each term

$$
\sum_{j_{1}, j_{2}, \ldots, j_{t-1}}\left(a_{i j_{1}}^{(p 1)} a_{j_{1} j_{2}}^{(p 2)} \cdots a_{j_{t-1} j}^{(p t)}\right)
$$

is the entry in the $(i, j)$-th position of $A_{p 1} A_{p 2} \cdots A_{p t}$. This completes the proof.

It should be noted that its proof of (1.3) is included for the sake of completeness, since some of the proofs below can be proved by using similar ideas. The same proofs were given in [8, Th.3.16] in greater generality for sets of matrices. In the present paper only the special case for usual spectral radius is presented with the same proof.

Proof of Huang's Inequality (1.3). Since the Hadamard product is commutative, we have

$$
\begin{aligned}
\left(A_{1} \circ A_{2} \circ \cdots \circ A_{k}\right)^{k} & =\left(A_{1} \circ A_{2} \circ \cdots \circ A_{k}\right)\left(A_{2} \circ \cdots A_{k} \circ A_{1}\right) \cdots\left(A_{k} \circ A_{1} \circ \cdots \circ A_{k-1}\right) \\
& \leq\left(A_{1} A_{2} \cdots A_{k}\right) \circ\left(A_{2} \cdots A_{k} A_{1}\right) \circ \cdots \circ\left(A_{k} A_{1} \cdots A_{k-1}\right)
\end{aligned}
$$

where the inequality follows from Lemma 2.2. By Lemma 2.1, we have

$$
\begin{aligned}
\rho^{k}\left(A_{1} \circ A_{2} \circ \cdots \circ A_{k}\right) & =\rho\left(\left(A_{1} \circ A_{2} \circ \cdots \circ A_{k}\right)^{k}\right) \\
& \leq \rho\left(\left(A_{1} A_{2} \cdots A_{k}\right) \circ\left(A_{2} \cdots A_{k} A_{1}\right) \circ \cdots \circ\left(A_{k} A_{1} \cdots A_{k-1}\right)\right) \\
& \leq \rho\left(A_{1} A_{2} \cdots A_{k}\right) \rho\left(A_{2} \cdots A_{k} A_{1}\right) \cdots \rho\left(A_{k} A_{1} \cdots A_{k-1}\right) \\
& =\rho^{k}\left(A_{1} A_{2} \cdots A_{k}\right) .
\end{aligned}
$$

In the last equality above we use the fact that

$$
\rho\left(A_{2} \cdots A_{k} A_{1}\right)=\rho\left(A_{3} A_{4} \cdots A_{2}\right)=\cdots=\rho\left(A_{k} A_{1} \cdots A_{k-1}\right)=\rho\left(A_{1} A_{2} \cdots A_{k}\right) .
$$

This completes the proof.

Denote by $\|A\|$ the spectral norm of $A \in M_{n}$, which equals to the largest singular value. The following interesting inequality is also due to Huang [6, Corollary 6]. We give a new proof.

Corollary 2.3. [6] Let $A, B \in M_{n}$ be nonnegative. Then

$$
\|A \circ B\| \leq \rho\left(A^{T} B\right)
$$


Proof. Note that $\rho\left(C^{T} C\right)=\|C\|^{2}$. Then

$$
\|A \circ B\|=\rho^{\frac{1}{2}}\left(\left(A^{T} \circ B^{T}\right)(A \circ B)\right)=\rho^{\frac{1}{2}}\left(\left(A^{T} \circ B^{T}\right)(B \circ A)\right) .
$$

By Lemma 2.1, we have

$$
\rho\left(\left(A^{T} \circ B^{T}\right)(B \circ A)\right) \leq \rho\left(\left(A^{T} B\right) \circ\left(B^{T} A\right)\right) \leq \rho\left(A^{T} B\right) \rho\left(B^{T} A\right)=\rho^{2}\left(A^{T} B\right) .
$$

This completes the proof.

The next inequality refines the inequality due to Huang [6]:

$$
\left\|A_{1} \circ A_{2} \circ \cdots \circ A_{k}\right\| \leq \rho^{\frac{1}{2}}\left(A_{1} A_{1}^{T} A_{2} A_{2}^{T} \cdots A_{k} A_{k}^{T}\right) .
$$

Proposition 2.4. Let $A_{1}, A_{2}, \ldots, A_{k} \in M_{n}$ be nonnegative matrices. Then

$$
\left\|A_{1} \circ A_{2} \circ \cdots \circ A_{k}\right\| \leq \rho^{\frac{1}{2}}\left(\left(A_{1} A_{1}^{T}\right) \circ\left(A_{2} A_{2}^{T}\right) \circ \cdots \circ\left(A_{k} A_{k}^{T}\right)\right) .
$$

Proof. Note that for any nonnegative square matrix $C, \rho\left(C^{T} C\right)=\|C\|^{2}$. Then

$$
\left\|A_{1} \circ A_{2} \circ \cdots \circ A_{k}\right\|=\rho^{\frac{1}{2}}\left(\left(A_{1} \circ A_{2} \circ \cdots \circ A_{k}\right)\left(A_{1} \circ A_{2} \circ \cdots \circ A_{k}\right)^{T}\right) .
$$

Since

$$
\left(A_{1} \circ A_{2} \circ \cdots \circ A_{k}\right)\left(A_{1} \circ A_{2} \circ \cdots \circ A_{k}\right)^{T}=\left(A_{1} \circ A_{2} \circ \cdots \circ A_{k}\right)\left(A_{1}^{T} \circ A_{2}^{T} \circ \cdots \circ A_{k}^{T}\right)
$$

by Lemma 2.2, we have

$$
\left(A_{1} \circ A_{2} \circ \cdots \circ A_{k}\right)\left(A_{1}^{T} \circ A_{2}^{T} \circ \cdots \circ A_{k}^{T}\right) \leq\left(A_{1} A_{1}^{T}\right) \circ\left(A_{2} A_{2}^{T}\right) \circ \cdots \circ\left(A_{k} A_{k}^{T}\right) .
$$

Therefore

$$
\begin{aligned}
\left\|A_{1} \circ A_{2} \circ \cdots \circ A_{k}\right\| & =\rho^{\frac{1}{2}}\left(\left(A_{1} \circ A_{2} \circ \cdots \circ A_{k}\right)\left(A_{1}^{T} \circ A_{2}^{T} \circ \cdots \circ A_{k}^{T}\right)\right) \\
& \leq \rho^{\frac{1}{2}}\left(\left(A_{1} A_{1}^{T}\right) \circ\left(A_{2} A_{2}^{T}\right) \circ \cdots \circ\left(A_{k} A_{k}^{T}\right)\right) .
\end{aligned}
$$

This completes the proof.

We need the following lemma whose proof can be found in [1, Lemma 1].

Lemma 2.5. [1] Let $A \in M_{n}$ be a positive matrix. Then $\rho(A)=\lim _{m \rightarrow \infty}\left(\operatorname{Tr} A^{m}\right)^{\frac{1}{m}}$.

Next, using Lemma 2.5 we prove the inequality which may be regarded as a Cauchy-Schwarz inequality for spectral radius of nonnegative matrices. This inequality is a consequence of a well known result for nonnegative matrices due to Elsner, Johnson and Silva [3], i.e.,

$$
\rho\left(A^{\left(\frac{1}{2}\right)} \circ B^{\left(\frac{1}{2}\right)}\right) \leq \rho(A)^{\frac{1}{2}} \rho(B)^{\frac{1}{2}},
$$

where $A^{(t)}$ denotes the Hadamard-Schur power. It should be noted that a new proof is included for the sake of completeness.

Proposition 2.6. Let $A, B \in M_{n}$ be nonnegative matrices. Then

$$
\rho(A \circ B) \leq[\rho(A \circ A)]^{\frac{1}{2}}[\rho(B \circ B)]^{\frac{1}{2}} .
$$


Proof. Without loss of generality we may assume that $A, B \in M_{n}$ are positive, since the spectral radius is continuous in matrices. Let $A=\left(a_{i j}\right), B=\left(b_{i j}\right) \in M_{n}$. For any positive integer $k$, we have

$$
\begin{aligned}
\operatorname{Tr}\left((A \circ B)^{2 k}\right) & =\sum_{i_{1}, i_{2}, \ldots, i_{2 k}}\left(a_{i_{1} i_{2}} b_{i_{1} i_{2}}\right)\left(a_{i_{2} i_{3}} b_{i_{2} i_{3}}\right) \cdots\left(a_{i_{2 k} i_{1}} b_{i_{2 k} i_{1}}\right) \\
& =\sum_{i_{1}, i_{2}, \ldots, i_{2 k}}\left(a_{i_{1} i_{2}} a_{i_{2} i_{3}} \cdots a_{i_{2 k} i_{1}}\right)\left(b_{i_{1} i_{2}} b_{i_{2 k} i_{1}} b_{i_{2} i_{3}} \cdots b_{i_{2 k} i_{1}}\right) \\
& \leq\left(\sum_{i_{1}, i_{2}, \ldots, i_{2 k}}\left(a_{i_{1} i_{2}} a_{i_{2} i_{3}} \cdots a_{i_{2 k} i_{1}}\right)^{2}\right)^{\frac{1}{2}}\left(\sum_{i_{1}, i_{2}, \ldots, i_{2 k}}\left(b_{i_{1} i_{2}} b_{i_{2} i_{3}} \cdots b_{i_{2 k} i_{1}}\right)^{2}\right)^{\frac{1}{2}} \\
& =\left(\sum_{i_{1}, i_{2}, \ldots, i_{2 k}} a_{i_{1} i_{2}}^{2} a_{i_{2} i_{3}}^{2} \cdots a_{i_{2 k} i_{1}}^{2}\right)^{\frac{1}{2}}\left(\sum_{i_{1}, i_{2}, \ldots, i_{2 k}} b_{i_{1} i_{2}}^{2} b_{i_{2} i_{3}}^{2} \cdots b_{i_{2 k} i_{1}}^{2}\right)^{\frac{1}{2}} \\
& =\left[\operatorname{Tr}(A \circ A)^{2 k}\right]^{\frac{1}{2}}\left[\operatorname{Tr}(B \circ B)^{2 k}\right]^{\frac{1}{2}} .
\end{aligned}
$$

The above inequality follows from the Cauchy-Schwarz inequality. Taking the $2 k$-th root, we have

$$
\left[\operatorname{Tr}\left((A \circ B)^{2 k}\right)\right]^{\frac{1}{2 k}} \leq\left[\operatorname{Tr}(A \circ A)^{2 k}\right]^{\frac{1}{4 k}}\left[\operatorname{Tr}(B \circ B)^{2 k}\right]^{\frac{1}{4 k}} .
$$

Taking the limit $k \rightarrow \infty$, and invoking Lemma 2.5 we obtain the inequality (2.2). This completes the proof.

Remark 2.7. Recall that Audenaert [1] proved

$$
\rho(A \circ B) \leq \rho^{\frac{1}{2}}((A \circ A)(B \circ B)) .
$$

We remark that the above inequality and the inequality (2.2) are not comparable in general. Here are two examples. Consider

$$
A=\left[\begin{array}{cc}
\sqrt{2} & \sqrt{2} \\
\sqrt{2} & \sqrt{2}
\end{array}\right], B=\left[\begin{array}{ll}
0 & 1 \\
0 & 0
\end{array}\right] .
$$

Then

$$
\rho^{\frac{1}{2}}((A \circ A)(B \circ B))=\sqrt{2}>0=[\rho(A \circ A)]^{\frac{1}{2}}[\rho(B \circ B)]^{\frac{1}{2}} .
$$

But for

$$
A=\left[\begin{array}{cc}
0 & 0 \\
0 & \sqrt{2}
\end{array}\right], B=\left[\begin{array}{ll}
1 & 0 \\
0 & 0
\end{array}\right]
$$

then

$$
\rho^{\frac{1}{2}}((A \circ A)(B \circ B))=0<\sqrt{2}=[\rho(A \circ A)]^{\frac{1}{2}}[\rho(B \circ B)]^{\frac{1}{2}} .
$$

Combining Lemma 2.2 and Proposition 2.6, we obtain the inequality due to Peperko [8].

Corollary 2.8. [8] Let $A, B \in M_{n}$ be nonnegative matrices. Then

$$
\rho(A \circ B) \leq \rho^{\frac{1}{2}}(A B \circ B A) \leq \rho^{\frac{1}{4}}(A B \circ A B) \rho^{\frac{1}{4}}(B A \circ B A) \leq \rho(A B) .
$$

Finally, we interpolate Huang's inequality (1.3) by proving 
Theorem 2.9. Let $A_{1}, A_{2}, \ldots, A_{k} \in M_{n}$ be nonnegative matrices. Then

$$
\begin{aligned}
\rho\left(A_{1} \circ A_{2} \circ \cdots \circ A_{k}\right) & \leq\left[\rho\left(A_{1} A_{2} \cdots A_{k}\right)\right]^{1-\frac{2}{k}}\left[\rho\left(\left(A_{1} \circ A_{1}\right)\left(A_{2} \circ A_{2}\right) \cdots\left(A_{k} \circ A_{k}\right)\right]^{\frac{1}{k}}\right. \\
& \leq \rho\left(A_{1} A_{2} \cdots A_{k}\right) .
\end{aligned}
$$

Proof. It suffices to prove this theorem for the case of positive matrices. Because of the continuity of the spectral radius, the theorem follows for the case of nonnegative matrices as well. Next, we assume that all the matrices $A_{1}, \ldots, A_{k}$ are positive.

Denote by $a_{i j}^{(t)}$ the entry of the matrix $A_{t}$ in the position $(i, j)$, for $t=1, \ldots, k$. Then

$$
\begin{aligned}
& \operatorname{Tr}\left(\left(A_{1} \circ A_{2} \circ \cdots \circ A_{k}\right)^{m k}\right)=\operatorname{Tr}\left[\left(\left(A_{1} \circ \cdots A_{k}\right)\left(A_{2} \circ \cdots \circ A_{1}\right) \cdots\left(A_{k} \circ \cdots \circ A_{k-1}\right)\right)^{m}\right] \\
& \leq \sum_{i_{1}, i_{2}, \ldots, i_{m k}}\left(a_{i_{1} i_{2}}^{(1)} a_{i_{1} i_{2}}^{(2)} \cdots a_{i_{1} i_{2}}^{(k)}\right)\left(a_{i_{2} i_{3}}^{(2)} \cdots a_{i_{2} i_{3}}^{(k)} a_{i_{2} i_{3}}^{(1)}\right) \cdots\left(a_{i_{m k} i_{1}}^{(k)} a_{i_{m k} i_{1}}^{(1)} \cdots a_{i_{m k} i_{1}}^{(k-1)}\right) \\
& =\sum_{i_{1}, i_{2}, \ldots, i_{m k}}^{(2)}\left[\left(a_{i_{1} i_{2}}^{(1)} a_{i_{2} i_{3}}^{(2)} \cdots a_{i_{m k} i_{1}}^{(k)}\right)\left(a_{i_{1} i_{2}}^{(2)} a_{i_{2} i_{3}}^{(3)} \cdots a_{i_{m k} i_{1}}^{(1)}\right)\right. \\
& \leq \sum_{i_{1}, i_{2}, \ldots, i_{m k}}\left(a_{i_{1} i_{2}}^{(1)} a_{i_{2} i_{3}}^{(2)} \cdots a_{i_{m k} i_{1}}^{(k)}\right)\left(a_{i_{1} i_{2}}^{(2)} a_{i_{2} i_{3}}^{(3)} \cdots a_{i_{m k} i_{1}}^{(1)}\right) \\
& \left.\left(a_{i_{1} i_{2}}^{(3)} a_{i_{2} i_{3}}^{(4)} \cdots a_{i_{m k} i_{1}}^{(2)}\right) \cdots\left(a_{i_{1} i_{2}}^{(k)} a_{i_{2} i_{3}}^{(1)} \cdots a_{i_{m k} i_{1}}^{(k-1)}\right)\right] \\
& \sum_{i_{1}, i_{2}, \ldots, i_{m k}}\left(a_{i_{1} i_{2}}^{(3)} a_{i_{2} i_{3}}^{(4)} \cdots a_{i_{m k} i_{1}}^{(2)}\right) \cdots \sum_{i_{1}, i_{2}, \ldots, i_{m k}}\left(a_{i_{1} i_{2}}^{(k)} a_{i_{2} i_{3}}^{(1)} \cdots a_{i_{m k} i_{1}}^{(k-1)}\right) .
\end{aligned}
$$

Note that

$$
\begin{aligned}
& \sum_{i_{1}, i_{2}, \ldots, i_{m k}}\left(a_{i_{1} i_{2}}^{(3)} a_{i_{2} i_{3}}^{(4)} \cdots a_{i_{m k} i_{1}}^{(2)}\right) \cdots \sum_{i_{1}, i_{2}, \ldots, i_{m k}}\left(a_{i_{1} i_{2}}^{(k)} a_{i_{2} i_{3}}^{(1)} \cdots a_{i_{m k} i_{1}}^{(k-1)}\right) \\
& =\operatorname{Tr}\left(\left(A_{3} A_{4} \cdots A_{2}\right)^{m}\right) \operatorname{Tr}\left(\left(A_{4} A_{5} \cdots A_{3}\right)^{m}\right) \cdots \operatorname{Tr}\left(\left(A_{k} A_{1} \cdots A_{k-1}\right)^{m}\right) .
\end{aligned}
$$

The expression $\sum_{i_{1}, i_{2}, \ldots, i_{m k}}\left(a_{i_{1} i_{2}}^{(1)} a_{i_{2} i_{3}}^{(2)} \cdots a_{i_{m k} i_{1}}^{(k)}\right)\left(a_{i_{1} i_{2}}^{(2)} a_{i_{2} i_{3}}^{(3)} \cdots a_{i_{m k} i_{1}}^{(1)}\right)$ is the standard Euclidean inner product of two nonnegative vectors in $\mathrm{R}^{n^{m k}}$, one with components $a_{i_{1} i_{2}}^{(1)} a_{i_{2} i_{3}}^{(2)} \cdots a_{i_{m k} i_{1}}^{(k)}$ and the other with components $a_{i_{1} i_{2}}^{(2)} a_{i_{2} i_{3}}^{(3)} \cdots a_{i_{m k} i_{1}}^{(1)}$. One sees that these two vectors have the same sets of components (which can be seen by performing a cyclic permutation on the indices $\left.i_{1}, i_{2}, \ldots, i_{m k}\right)$. Thus both vectors have the same Euclidean norm. Applying the Cauchy-Schwarz inequality then $\begin{aligned} \text { gives } & \sum_{\quad}\left(a_{i_{1} i_{2}}^{(1)} a_{i_{2} i_{3}}^{(2)} \cdots a_{i_{m k} i_{1}}^{(k)}\right)\left(a_{i_{1} i_{2}}^{(2)} a_{i_{2} i_{3}}^{(3)} \cdots a_{i_{m k} i_{1}}^{(1)}\right) \\ \leq & \sum_{i_{1}, i_{2}, \ldots, i_{m k}}^{(1)}\left(\left(a_{i_{1} i_{2}}^{(1)}\right)^{2}\left(a_{i_{2} i_{3}}^{(2)}\right)^{2} \cdots\left(a_{i_{m k} i_{1}}^{(k)}\right)^{2}\right)=\operatorname{Tr}\left[\left(\left(A_{1} \circ A_{1}\right)\left(A_{2} \circ A_{2}\right) \cdots\left(A_{k} \circ A_{k}\right)\right)^{m}\right] .\end{aligned}$

This proves that the following inequality holds for any positive integer $\mathrm{m}$ : $\operatorname{Tr}\left(\left(A_{1} \circ A_{2} \circ \cdots \circ A_{k}\right)^{m k}\right)$

$$
\begin{aligned}
& \leq \operatorname{Tr}\left(\left(A_{3} A_{4} \cdots A_{2}\right)^{m}\right) \operatorname{Tr}\left(\left(A_{4} A_{5} \cdots A_{3}\right)^{m}\right) \cdots \operatorname{Tr}\left(\left(A_{k} A_{1} \cdots A_{k-1}\right)^{m}\right) \\
& \quad \times \operatorname{Tr}\left[\left(\left(A_{1} \circ A_{1}\right)\left(A_{2} \circ A_{2}\right) \cdots\left(A_{k} \circ A_{k}\right)\right)^{m}\right] .
\end{aligned}
$$

Taking the $m k$-th root on the above inequality, taking the limit $m \rightarrow \infty$, we have

$$
\begin{gathered}
\rho\left(A_{1} \circ A_{2} \circ \cdots \circ A_{k}\right) \leq \rho^{\frac{1}{k}}\left(A_{3} A_{4} \cdots A_{2}\right) \rho^{\frac{1}{k}}\left(A_{4} A_{5} \cdots A_{3}\right) \cdots \rho^{\frac{1}{k}}\left(A_{k} A_{1} \cdots A_{k-1}\right) \\
\times\left[\rho\left(\left(A_{1} \circ A_{1}\right)\left(A_{2} \circ A_{2}\right) \cdots\left(A_{k} \circ A_{k}\right)\right]^{\frac{1}{k}} .\right.
\end{gathered}
$$


Using the fact

$$
\rho\left(A_{3} A_{4} \cdots A_{2}\right)=\rho\left(A_{4} A_{5} \cdots A_{3}\right)=\cdots=\rho\left(A_{k} A_{1} \cdots A_{k-1}\right)=\rho\left(A_{1} A_{2} \cdots A_{k}\right),
$$

we obtain the first inequality.

On the other hand, by Lemma 2.2,

$$
\left(A_{1} \circ A_{1}\right)\left(A_{2} \circ A_{2}\right) \cdots\left(A_{k} \circ A_{k}\right) \leq\left(A_{1} A_{2} \cdots A_{k}\right) \circ\left(A_{1} A_{2} \cdots A_{k}\right) .
$$

Applying Lemma 2.1 we have

$$
\left[\rho\left(\left(A_{1} \circ A_{1}\right)\left(A_{2} \circ A_{2}\right) \cdots\left(A_{k} \circ A_{k}\right)\right)\right]^{\frac{1}{k}} \leq \rho^{\frac{2}{k}}\left(\left(A_{1} A_{2} \cdots A_{k}\right)\right) .
$$

This completes the proof.

Remark 2.10. The inequality (1.1) corresponds to the case $k=2$ of Theorem 2.9.

Acknowledgement. The authors are grateful to the two referees for their helpful and constructive suggestions. In addition, the second author would like to thank Professor Xingzhi Zhan for his encouragement. This research was supported by the NSFC grant 11371145 and Anhui Provincial Natural Science Foundation (1408085MA08, 1308085QF117).

\section{REFERENCES}

1. K.M.R. Audenaert, Spectral radius of Hadamard product versus conventional product for non-negative matrices, Linear Algebra Appl. 432 (2010), 366-368.

2. R. Drnovšsek and A. Peperko, Inequalities for the Hadamard Weighted Geo- metric Mean of Positive Kernel Operators on Banach Function Spaces, Positivity 10 (2006), 613-626.

3. L. Elsner, C.R. Johnson and J.A. Dias Da Silva, The Perron root of a weighted geometric mean of nonnegative matrices, Linear Multilinear Algebra, 24 (1998), 1-13.

4. R.A. Horn and C.R. Johnson, Topics in Matrix Analysis, Cambridge University Press, 1985.

5. R.A. Horn and F. Zhang, Bounds on the spectral radius of a Hadamard product of nonnegative or positive semidefinite matrices, Electron. J. Linear Algebra 20 (2010), 90-94.

6. Z. Huang, On the spectral radius and the spectral norm of Hadamard products of nonnegative matrices, Linear Algebra Appl. 434 (2010), 457-462.

7. A. Peperko, Inequalities for the spectral radius of non-negative functions, Positivity $\mathbf{1 3}$ (2009), 255-272.

8. A. Peperko, Bounds on the generalzied and the joint spectral radius of Hadamard products of bounded sets of positive operators on sequence spaces, Linear Algebra Appl. 437 (2012), 189-201.

9. A.R. Schep, Bounds on the spectral radius of Hadamard products of positive operators on $\ell_{p}$-spaces, Electron. J. Linear Algebra 22 (2011), 443-447.

10. X. Zhan, Unsolved matrix problems, Talk given at Advance Workshop on Trends and Developments in Linear algebra, ICTP, Trieste, Italy, July 6-10, 2009.

11. X. Zhan, Matrix Theory, Graduate Studies in Mathematics 147, American Mathematical Society, Providence, R.I., 2013.

School of Mathematical Sciences, Huaibei Normal University, Huaibei 235000, CHINA.

E-mail address: djcmaths@163.com; zhangyunmaths@163.com 